\title{
Embedding of Besov Spaces and the Volterra Integral Operator
}

\author{
Dan Qu $\left(\mathbb{D},{ }^{1}\right.$ Xiangling Zhu $(\mathbb{D})^{2}$ and Ruishen Qian $\mathbb{D}^{3}$ \\ ${ }^{1}$ Faculty of Information Technology, Macau University of Science and Technology, Avenida Wai Long, Macau, China \\ ${ }^{2}$ University of Electronic Science and Technology of China, Zhongshan Institute, Zhongshan 528402, Guangdong, China \\ ${ }^{3}$ School of Mathematics and Statistics, Lingnan Normal University, Zhanjiang 524048, Guangdong, China
}

Correspondence should be addressed to Xiangling Zhu; jyuzxl@163.com

Received 20 February 2021; Accepted 27 March 2021; Published 13 April 2021

Academic Editor: Efthymios G. Tsionas

Copyright (c) 2021 Dan Qu et al. This is an open access article distributed under the Creative Commons Attribution License, which permits unrestricted use, distribution, and reproduction in any medium, provided the original work is properly cited.

The boundedness and compactness of the inclusion mapping from Besov spaces to tent spaces are studied in this paper. Meanwhile, the boundedness, compactness, and essential norm of the Volterra integral operator $T_{g}$ from Besov spaces to a class of general function spaces are also investigated.

\section{Introduction}

Let $\mathscr{D}$ be the unit disk in the complex plane $\mathbb{C}$ and $H(\mathscr{D})$ be the class of functions analytic in $\mathscr{D}$. For $1<p<\infty$, the Besov space, denoted by $B_{p}$, is the space of all functions $f \in H(\mathscr{D})$ satisfying

$$
\|f\|_{B_{p}}^{p}=|f(0)|^{p}+\int_{\mathscr{D}}\left|f^{\prime}(z)\right|^{p}\left(1-|z|^{2}\right)^{p-2} \mathrm{~d} A(z)<\infty .
$$

Let $0<p<\infty,-2<q<\infty$, and $0 \leq s<\infty$. The space $F(p, q, s)$ is the space consisting of all $f \in H(\mathscr{D})$ such that

$$
\begin{aligned}
\|f\|_{F(p, q, s)}^{p}= & |f(0)|^{p}+\sup _{a \in \mathscr{D}} \int_{\mathscr{D}}\left|f^{\prime}(z)\right|^{p}\left(1-|z|^{2}\right)^{q} \\
& \left(1-\left|\sigma_{a}(z)\right|^{2}\right)^{s} \mathrm{~d} A(z)<\infty,
\end{aligned}
$$

where $\sigma_{a}(z)=((a-z) /(1-\bar{a} z))$. This space was first introduced by Zhao in [1]. $F(2,0, s)$ is the $Q_{s}$ space (see [2]). $F(2,0,1)$ is the BMOA space. $F(p, \alpha, 0)$ is called the Dirichlet-type space, denoted by $\mathscr{D}_{\alpha}^{p}$. In particular, $F(p, p-$ $2,0)$ is the Besov space $B_{p} . F(p, p, 0)$ is just the classical Bergman space $A^{p}$. When $s>1$, from [1], we see that $F(p, p-2, s)$ is equivalent to the Bloch space, denoted by $\mathscr{B}$, which consists of all $f \in H(\mathscr{D})$ such that

$$
\|f\|_{\mathscr{B}}=|f(0)|+\sup _{z \in \mathscr{D}}\left(1-|z|^{2}\right)\left|f^{\prime}(z)\right|<\infty .
$$

For $1<q<\infty$ and $0<s, t<\infty$, let $\mathscr{L} \mathscr{F}(q, q-2, s, t)$ denote the space of all $f \in H(\mathscr{D})$ such that

$$
\begin{aligned}
\|f\|_{L o g}^{q}= & \sup _{a \in \mathscr{D}} \frac{1}{\left(\log \left(2 / 1-|a|^{2}\right)\right)^{t}} \int_{\mathscr{D}}\left|f^{\prime}(z)\right|^{q}\left(1-|z|^{2}\right)^{q-2} \\
& \left(1-\left|\sigma_{a}(z)\right|^{2}\right)^{s} \mathrm{~d} A(z)<\infty .
\end{aligned}
$$

The norm for $f \in \mathscr{L} \mathscr{F}(q, q-2, s, t)$ is given by $\|f\|_{\mathscr{L} \mathscr{F}(q, q-2, s, t)}=|f(0)|+\|f\|_{\text {Log }}$. The space $\mathscr{L} \mathscr{F}(q, q-2$, $s, t)$ is a Banach space when $q>1$.

The Volterra integral operator $T_{g}$ was introduced by Pommerenke in [3]. Here,

$$
T_{g} f(z)=\int_{0}^{z} f(w) g^{\prime}(w) \mathrm{d} w, \quad f \in H(\mathscr{D}) .
$$

In [3], Pommerenke showed that $T_{g}$ is bounded on $H^{2}$ if and only if $g \in$ BMOA. Aleman and Siskakis showed that $T_{g}$ is bounded on $H^{p}(p \geq 1)$ if and only if $g \in$ BMOA in [4]. In [5], Aleman and Siskakis proved that $T_{g}: A^{p} \longrightarrow A^{p}$ is bounded (compact) if and only if $g \in \mathscr{B}\left(g \in \mathscr{B}_{0}\right)$. Recently, the operator $T_{g}$ has been receiving much attention. See [4-18] and the references therein for more study of the operator $T_{g}$. 
For any arc $I \subseteq \partial \mathbb{D}$, the boundary of $\mathscr{D}$, let $|I|=(1 / 2 \pi) \int_{I}|\mathrm{~d} \zeta|$ denote the normalized length of $I$ and $S(I)$ be the Carleson box defined by

$$
S(I)=\left\{z \in \mathscr{D}: 1-|I| \leq|z|<1, \frac{z}{|z|} \in I\right\} .
$$

Let $0<s<\infty$ and $\mu$ be a positive Borel measure on $\mathscr{D}$. We say that $\mu$ is a $\alpha$-Carleson measure if

$$
\|\mu\|_{\mathrm{CM}_{\alpha}}=\sup _{I \subset \partial \mathscr{D}} \frac{\mu(S(I))}{|I|^{\alpha}}<\infty .
$$

When $\alpha=1$, it gives the classical Carleson measure. $\mu$ is said to be a vanishing $\alpha$-Carleson measure if $\lim _{|I| \longrightarrow 0}(\mu(S$ $\left.(I)) /|I|^{\alpha}\right)=0$. The Carleson measure is very useful in the theory of function spaces and operator theory. The famous embedding theorem says that the inclusion mapping $I_{d}: H^{2} \longrightarrow L^{2}(\mathrm{~d} \mu)$ is bounded if and only if $\mu$ is a Carleson measure (see [19]). See [7, 20] for the study of the inclusion mapping $I_{d}: B_{p} \longrightarrow L^{p}(\mathrm{~d} \mu)$.

Let $0<s, q<\infty, 0 \leq t<\infty$, and $\mu$ be a positive Borel measure on $\mathbb{D}$. Let $\mathscr{T}_{s, t}^{q}(\mu)$ denote the space of all $\mu$-measurable functions $f$ such that (see, e.g., [21])

$$
\sup _{I \subset \partial \mathscr{D}} \frac{1}{|I|^{S}(\log (2 /|I|))^{t}} \int_{S(I)}|f(z)|^{q} \mathrm{~d} \mu(z)<\infty .
$$

The tent space $\mathscr{T}_{s, t}^{q}(\mu)$ was introduced by Liu et al. in [21]. When $t=0, \mathscr{T}_{s, t}^{q}(\mu)$ will be denoted by $\mathscr{T}_{s}^{q}(\mu)$ for the simplicity. In [21], Liu et al. studied the embedding of some Möbius invariant spaces, such as the Bloch space and the $Q_{p}$ space, into $\mathscr{T}_{s, t}^{2}$.

In [12], Pau and Zhao showed that the inclusion mapping $I_{d}: F(p, p-2, s) \longrightarrow \mathscr{T}_{s}^{p}(\mu)$ is bounded if and only if $\mu$ is a $p$-logarithmic $s$-Carleson measure. In [9], Li et al. proved that the inclusion mapping $I_{d}: \mathscr{D}_{p-1}^{p} \longrightarrow \mathscr{T}_{s}^{p}(\mu)$ is bounded if and only if $\mu$ is a $(s+1)$-Carleson measure. In [14], Qian and $\mathrm{Li}$ proved that the inclusion mapping $I_{d}: B_{p} \longrightarrow \mathscr{T}_{s}^{q}(\mu)$ is bounded (resp. compact) if and only if $\mu$ is a $q(1-(1 / p))$-logarithmic $s$-Carleson measure (resp. vanishing $q(1-(1 / p))$-logarithmic $s$-Carleson measure) under the assumption that $1<p<q<\infty$ and $0<s<\infty$.

Motivated by $[14,21]$, in this paper, we study the boundedness and compactness of the inclusion mapping $I_{d}: B_{p} \longrightarrow \mathscr{T}_{s, q(1-(1 / p))}^{q}(\mu)$. More precisely, we show that $I_{d}: B_{p} \longrightarrow \mathscr{T}_{s, q(1-(1 / p))}^{q_{q} q(1-(1 / p))}(\mu)$ is bounded (resp. compact) if and only if $\mu$ is an $s$-Carleson measure (resp. vanishing $s$-Carleson measure) under the assumption that $1<p<q<\infty$ and $0<s<\infty$. As an application, we study the boundedness of the operator $T_{g}: B_{p} \longrightarrow \mathscr{L F}(q, q$ $-2, s, q-(q / p))$. Moreover, the compactness and essential norm of the operator $T_{g}: B_{p} \longrightarrow \mathscr{L} \mathscr{F}(q, q-2, s, q-(q / p))$ are also investigated.

In this paper, the symbol $f \approx g$ means that $f \leqslant g \leqslant f$. We say that $f \leqslant g$ if there exists a constant $C$ such that $f \leq C g$.

\section{Embedding the Besov Space $B_{p}$ into $\mathscr{T}_{s, t}^{q}(\boldsymbol{\mu})$}

We need the following equivalent description of $\alpha$-Carleson measure (see Lemma 2.2 in [12]).

Lemma 1. Let $0<\alpha, t<\infty$ and $\mu$ be a positive Borel measure on $\mathbb{D}$. Then, $\mu$ is an $\alpha$-Carleson measure if and only if

$$
\sup _{a \in \mathbb{D}} \int_{\mathbb{D}} \frac{\left(1-|a|^{2}\right)^{t}}{|1-\bar{a} z|^{\alpha+t}} \mathrm{~d} \mu(z)<\infty .
$$

Moreover,

$$
\|\mu\|_{\mathrm{CM}_{\alpha}} \approx \sup _{a \in \mathbb{D}} \int_{\mathbb{D}} \frac{\left(1-|a|^{2}\right)^{t}}{|1-\bar{a} z|^{\alpha+t}} \mathrm{~d} \mu(z) .
$$

Using Lemma 3.10 in [22], we can easily obtain the following result.

Lemma 2. Let $1<p<\infty$ and $w \in \mathscr{D}$. Set

$$
f_{w}(z)=\left(\frac{1}{\log \left(2 / 1-|w|^{2}\right)}\right)^{1 / p} \log \frac{2}{1-\bar{w} z}, \quad z \in \mathscr{D} .
$$

Then, $f_{w} \in B_{p}$.

Theorem 1. Let $1<p<q<\infty, 0<s<\infty$, and $\mu$ be a positive Borel measure on $\mathscr{D}$. Then, the inclusion mapping $I_{d}: B_{p} \longrightarrow$ $\mathscr{T}_{s, q(1-(1 / p))}^{q}(\mu)$ is bounded if and only if $\mu$ is an $s$-Carleson measure.

Proof. First, we assume that $I_{d}: B_{p} \longrightarrow \mathscr{T}_{s, q(1-(1 / p))}^{q}(\mu)$ is bounded. For any given arc $I \subseteq \partial \mathscr{D}$, set $a=(1-|I|) \eta$, and $\eta$ is the center point of $I$. It is easy to see that

$$
|1-\bar{a} z| \approx 1-|a|^{2} \approx|I|, \quad z \in S(I) .
$$

Let

$$
f_{a}(z)=\left(\frac{1}{\log \left(2 / 1-|a|^{2}\right)}\right)^{1 / p} \log \frac{2}{1-\bar{a} z} .
$$

By Lemma 2, we see that $f_{a} \in B_{p}$. From the boundedness of $I_{d}: B_{p} \longrightarrow \mathscr{T}_{s, q(1-(1 / p))}^{q}(\mu)$, we have

$$
\begin{aligned}
\left\|f_{a}\right\|_{\mathscr{T}_{s}^{q}(\mu)}^{q}= & \sup _{I \subset \partial \mathscr{D}} \frac{1}{|I|^{s}(\log (2 /|I|))^{q(1-(1 / p))}} \\
& \cdot \int_{S(I)}\left|f_{a}(z)\right|^{q} \mathrm{~d} \mu(z)<\infty .
\end{aligned}
$$

By the fact that $\left|f_{a}(z)\right| \approx(\log (2 /|I|))^{1-(1 / p)}$ when $z \in S(I)$, we get

$$
\sup _{I \subset \partial D} \frac{\mu(S(I))}{|I|^{s}}<\infty
$$

Hence, $\mu$ is an $s$-Carleson measure. 
Conversely, assume that $\mu$ is an $s$-Carleson measure. Let $f \in B_{p}$. For any given $\operatorname{arc} I \subseteq \partial \mathscr{D}$, set $w=(1-|I|) \eta$, and $\eta$ is the center point of $I$. Then,

$$
\begin{aligned}
\frac{1}{|I|^{S}(\log (2 /|I|))^{q(1-(1 / p))}} \int_{S(I)}|f(z)|^{q} \mathrm{~d} \mu(z) \lesssim & \frac{1}{|I|^{s}(\log (2 /|I|))^{q(1-(1 / p))}} \int_{S(I)}|f(z)-f(w)|^{q} \mathrm{~d} \mu(z) \\
& +\frac{1}{|I|^{s}(\log (2 /|I|))^{q(1-(1 / p))}} \int_{S(I)}|f(w)|^{q} \mathrm{~d} \mu(z) \\
& =A+B,
\end{aligned}
$$

where

$$
\begin{aligned}
& A=\frac{1}{|I|^{S}(\log (2 /|I|))^{q(1-(1 / p))}} \int_{S(I)}|f(z)-f(w)|^{q} \mathrm{~d} \mu(z), \\
& B=\frac{1}{|I|^{s}(\log (2 /|I|))^{q(1-(1 / p))}} \int_{S(I)}|f(w)|^{q} \mathrm{~d} \mu(z) .
\end{aligned}
$$

Since

$$
|f(w)| \lesssim\left(\log \frac{2}{1-|w|^{2}}\right)^{1-(1 / p)}\|f\|_{B_{p}} \lesssim\left(\log \frac{2}{|I|}\right)^{1-(1 / p)}\|f\|_{B_{p}}
$$

we get

$$
B \lesssim \frac{\mu(S(I))}{|I|^{s}}\|f\|_{B_{p}}^{q} \lesssim\|f\|_{B_{p}}^{q}
$$

Now, we turn to estimate $A$. By Theorem 1 in [7], we see that $\mu$ is an $s$-Carleson measure if and only if $I_{d}: D_{p-2+(p s / q)}^{p} \longrightarrow L^{q}(\mu)$ is bounded. Note that

$$
f \in B_{p} \subseteq D_{p-2+(p s / q)}^{p} \text {. }
$$

Then,

$$
A \lesssim\left(1-|w|^{2}\right)^{s} \int_{S(I)}\left|\frac{f(z)-f(w)}{(1-\bar{w} z)^{(2 s / q)}}\right|^{q} \mathrm{~d} \mu(z)
$$

$$
\leq\left(\left(1-\left.\right|^{2}\right)^{(p s / q)} \int_{\mathscr{D}}\left|\left(\frac{f(z)-f(w)}{(1-\bar{w} z)^{(2 s / q)}}\right)^{\prime}\right|^{p}\right.
$$

$$
\left.\cdot\left(1-|z|^{2}\right)^{p-2+(p s / q)} \mathrm{d} A(z)\right)^{(q / p)} .
$$

Since

$$
\left(\frac{f(z)-f(w)}{(1-\bar{w} z)^{(2 s / q)}}\right)^{\prime}=\frac{f^{\prime}(z)(1-\bar{w} z)^{(2 s / q)}+\bar{w}(2 s / q)(f(z)-f(w))(1-\bar{w} z)^{(2 s / q)-1}}{(1-\bar{w} z)^{(4 s / q)}}
$$

we deduce that $A \lesssim\left(W_{1}+W_{2}\right)^{(q / p)}$, where

$$
\begin{gathered}
W_{1}=\left(1-|w|^{2}\right)^{(p s / q)} \int_{\mathscr{D}|1-\bar{w} z|^{(2 p s / q)}} \frac{\left|f^{\prime}(z)\right|^{p}}{\left.\left.|1-| z\right|^{2}\right)^{p-2+(p s / q)} \mathrm{d} A(z),} \\
W_{2}=\left(1-|w|^{2}\right)^{(p s / q)} \int_{\mathscr{D}|1-\bar{w} z|^{(2 p s / q)+p}} \frac{|f(z)-f(w)|^{p}}{\left.\left.|1-| z\right|^{2}\right)^{p-2+(p s / q)} \mathrm{d} A(z) .}
\end{gathered}
$$

Since

$$
\frac{\left(1-|w|^{2}\right)\left(1-|z|^{2}\right)}{|1-\bar{w} z|^{2}}=\left(1-\left|\sigma_{w}(z)\right|^{2}\right) \leq 1,
$$

we get that

$$
W_{1} \lesssim\|f\|_{B_{p}}^{p} .
$$

Making the change of variable $\eta=\sigma_{w}(z)$ and combining with Proposition 4.2 in [22], we have 


$$
\begin{aligned}
W_{2} & =\left(1-|w|^{2}\right)^{(p s / q)} \int_{\mathscr{D}} \frac{\left|\left(f^{\circ} \sigma_{w}\right)(\eta)-\left(f^{\circ} \sigma_{w}\right)(0)\right|^{p}}{\left|1-\bar{w} \sigma_{w}(\eta)\right|^{(2 p s / q)+p}}\left(1-\left|\sigma_{w}(\eta)\right|^{2}\right)^{p-2+(p s / q)} \times \frac{\left(1-|w|^{2}\right)^{2}}{|1-\bar{w} \eta|^{4}} \mathrm{~d} A(\eta) \\
& =\int_{\mathscr{D}}\left|\left(f^{\circ} \sigma_{w}\right)(\eta)-\left(f^{\circ} \sigma_{w}\right)(0)\right|^{p} \frac{\left(1-|\eta|^{2}\right)^{p-2+(p s / q)}}{|1-\bar{w} \eta|^{p}} \mathrm{~d} A(\eta) \\
& \leq \int_{\mathscr{D}}\left|\left(f^{\circ} \sigma_{w}\right)^{\prime}(\eta)\right|^{p} \frac{\left(1-|\eta|^{2}\right)^{2 p-2+(p s / q)}}{|1-\bar{w} \eta|^{p}} \mathrm{~d} A(\eta) \\
& \leq \int_{\mathscr{D}}\left|f^{\prime}\left(\sigma_{w}(\eta)\right)\right|^{p}\left(1-\left|\sigma_{w}(\eta)\right|^{2}\right)^{p} \frac{\left(1-|\eta|^{2}\right)^{p-2+(p s / q)}}{|1-\bar{w} \eta|^{p}} \mathrm{~d} A(\eta) \\
& \leq \int_{\mathscr{D}}\left|f^{\prime}(z)\right|^{p}\left(1-|z|^{2}\right)^{p} \frac{\left(1-\left|\sigma_{w}(z)\right|^{2}\right)^{p-2+(p s / q)}}{\left|1-\bar{w} \sigma_{w}(z)\right|^{p}} \frac{\left(1-|w|^{2}\right)^{2}}{|1-\bar{w} z|^{4}} \mathrm{~d} A(z) \\
& \leq \int_{\mathscr{D}}\left|f^{\prime}(z)\right|^{p} \frac{\left(1-|w|^{2}\right)^{(p s / q)}\left(1-|z|^{2}\right)^{2 p-2+(p s / q)}}{|1-\bar{w} z|^{p+(2 p s / q)}} \mathrm{d} A(z) \\
& \leq\|f\|_{B_{p}}^{p} .
\end{aligned}
$$

Therefore,

$$
\sup _{I \subset \partial D} \frac{1}{|I|^{s}(\log (2 /|I|))^{q(1-(1 / p))}} \int_{S(I)}|f(z)|^{q} \mathrm{~d} \mu(z) \leq\|f\|_{B_{p}}^{q},
$$

which implies the desired result. The proof is completed.

We say that the inclusion mapping $I_{d}: B_{p} \longrightarrow \mathscr{T}_{s, q(1-(1 / p))}^{q}$ $(\mu)$ is compact if

$$
\lim _{n \longrightarrow \infty} \frac{1}{|I|^{s}(\log (2 /|I|))^{q(1-(1 / p))}} \int_{S(I)}\left|f_{n}(z)\right|^{q} \mathrm{~d} \mu(z)=0
$$

whenever $I \subset \partial \mathbb{D}$ and $\left\{f_{n}\right\}$ is a bounded sequence in $B_{p}$ that converges to 0 uniformly on compact subsets of $\mathbb{D}$.

Theorem 2. Let $1<p<q<\infty$ and $0<s<\infty$. Let $\mu$ be a nonnegative Borel measure on $\mathbb{D}$ such that point evaluation is a bounded functional on $\mathscr{T}_{s, q(1-(1 / p))}^{q}(\mu)$. Then, the inclusion mapping $I_{d}: B_{p} \longrightarrow \mathscr{T}_{s, q(1-(1 / p))}^{q}(\mu)$ is compact if and only if $\mu$ is a vanishing s-Carleson measure.

Proof. First, we assume that $I_{d}: B_{p} \longrightarrow \mathscr{T}_{s, q(1-(1 / p))}^{q}(\mu)$ is compact. Let $\left\{I_{k}\right\}$ be a sequence arc with $\lim _{k \longrightarrow \infty}, q\left(I_{k} \mid=0\right.$. Set $a_{k}=\left(1-\left|I_{k}\right|\right) \eta_{k}$, where $\eta_{k}$ is the midpoint of arc $I_{k}$. Take

$$
f_{k}(z)=\left(\frac{1}{\log \left(2 / 1-\left|a_{k}\right|^{2}\right)}\right)^{1 / p} \log \frac{2}{1-\overline{a_{k}} z} .
$$

We see that $f_{k} \in B_{p}$, and $\left\{f_{k}\right\}$ converges to 0 uniformly on compact subsets of $\mathscr{D}$ when $k \longrightarrow \infty$. Then, we get

$$
\frac{\mu\left(S\left(I_{k}\right)\right)}{\left|I_{k}\right|^{s}} \lesssim \frac{1}{\left|I_{k}\right|^{s}\left(\log \left(2 /\left|I_{k}\right|\right)\right)^{q(1-(1 / p))}} \int_{S\left(I_{k}\right)}\left|f_{k}(z)\right|^{q} \mathrm{~d} \mu(z) \longrightarrow 0,
$$

as $k \longrightarrow \infty$, which implies that $\mu$ is a vanishing $s$-Carleson measure.

Conversely, assume that $\mu$ is a vanishing $s$-Carleson measure. From [12], we see that

$$
\left\|\mu-\mu_{r}\right\|_{\mathrm{CM}_{s}} \longrightarrow 0, \quad r \longrightarrow 1 .
$$

Here, $\mu_{r}(z)=\mu(z)$ for $|z|<r$ and $\mu_{r}(z)=0$ for $r \leq|z|<1$. Let $\left\|f_{k}\right\|_{B_{p}} \leq 1$ and $\left\{f_{k}\right\}$ converge to 0 uniformly on compact subsets of $\mathscr{D}^{p}$. Then,

$$
\begin{aligned}
& \frac{1}{|I|^{s}(\log (2 /|I|))^{q(1-(1 / p))}} \int_{S(I)}\left|f_{k}(z)\right|^{q} \mathrm{~d} \mu(z) \\
& \leqslant \frac{1}{|I|^{s}(\log (2 /|I|))^{q(1-(1 / p))}} \int_{S(I)}\left|f_{k}(z)\right|^{q} \mathrm{~d} \mu_{r}(z) \\
& \quad+\frac{1}{|I|^{s}(\log (2 /|I|))^{q(1-(1 / p))}} \int_{S(I)}\left|f_{k}(z)\right|^{q} \mathrm{~d}\left(\mu-\mu_{r}\right)(z) \\
& \leqslant \frac{1}{|I|^{s}(\log (2 /|I|))^{q(1-(1 / p))}} \int_{S(I)}\left|f_{k}(z)\right|^{q} \mathrm{~d} \mu_{r}(z)+\left\|\mu-\mu_{r}\right\|_{\mathrm{CM}_{s}}\left\|f_{k}\right\|_{B_{p}}^{q} \\
& \lesssim \frac{1}{|I|^{s}(\log (2 /|I|))^{q(1-(1 / p))}} \int_{S(I)}\left|f_{k}(z)\right|^{q} \mathrm{~d} \mu_{r}(z)+\left\|\mu-\mu_{r}\right\|_{\mathrm{CM}_{s} .}
\end{aligned}
$$

Letting $k \longrightarrow \infty$ and then $r \longrightarrow 1$, we have

$$
\lim _{k \longrightarrow \infty}\left\|f_{k}\right\|_{\mathscr{T}_{s, q(1-(1 / p))}^{q}(\mu)}=0 \text {. }
$$


Therefore, $I_{d}: B_{p} \longrightarrow \mathscr{T}_{s, q(1-(1 / p))}^{q}(\mu)$ is compact. The proof is completed.

3. Volterra Integral Operator $T_{g}: B_{p} \longrightarrow \mathscr{L} \mathscr{F}$ $(q, q-2, s, q-(q / p))$

Lemma 3. Let $1<q<\infty$ and $0<s, t<\infty$. Then, $f \in \mathscr{L} \mathscr{F}(q, q-2, s, t)$ if and only if

$$
\sup _{I \subset \partial \mathscr{D}} \frac{1}{|I|^{s}(\log (2 /|I|))^{t}} \int_{S(I)}\left|f^{\prime}(z)\right|^{q}\left(1-|z|^{2}\right)^{q-2+s} \mathrm{~d} A(z)<\infty .
$$

Proof. The proof is similar to that of Proposition 1 in [15]. Thus, we omit the details of the proof.

Theorem 3. Let $1<p<q<\infty$ and $0<s<\infty$. Then, $T_{g}: B_{p}$ $\longrightarrow \mathscr{L} \mathscr{F}(q, q-2, s, q-(q / p))$ is bounded if and only if $g \in F(q, q-2, s)$

Proof. Assume that $T_{g}: B_{p} \longrightarrow \mathscr{L} \mathscr{F}(q, q-2, s, q-(q / p))$ is bounded. For any fixed arc $I \subseteq \partial \mathscr{D}$, let $e^{i \theta}$ denote the center of $I$ and $a=(1-|I|) e^{i \theta}$. Set

$$
f_{a}(z)=\left(\frac{1}{\log \left(2 / 1-|a|^{2}\right)}\right)^{1 / p} \log \frac{2}{1-\bar{a} z}
$$

By Lemma 2, we have $f_{a} \in B_{p}$ for $1<p<\infty$. In addition, it is easy to see that

$$
\begin{aligned}
& |1-\bar{a} z| \approx 1-|a|=|I|, \\
& \left|f_{a}(z)\right| \approx\left(\log \frac{2}{|I|}\right)^{(1-1 / p)},
\end{aligned}
$$

when $z \in S(I)$. By the boundedness of $T_{g}: B_{p} \longrightarrow \mathscr{L} \mathscr{F}(q, q-2, s, q-(q / p))$, we get

$$
\begin{aligned}
\infty & >\left\|T_{g} f_{a}\right\|_{\mathscr{L} \mathscr{F}(q, q-2, s, q-(q / p))}^{q} \\
& \geq \frac{1}{|I|^{s}(\log (2 /|I|))^{q(1-1 / p)}} \int_{S(I)}\left|f_{a}(z)\right|^{q}\left|g^{\prime}(z)\right|^{q} \\
& \left(1-|z|^{2}\right)^{q-2+s} \mathrm{~d} A(z) \\
& \approx \frac{1}{|I|^{s}} \int_{S(I)}\left|g^{\prime}(z)\right|^{q}\left(1-|z|^{2}\right)^{q-2+s} \mathrm{~d} A(z),
\end{aligned}
$$

which implies that $g \in F(q, q-2, s)$ by [1].

Conversely, suppose that $g \in F(q, q-2, s)$. By [1], we see that $\mathrm{d} \mu_{g}(z)=\left|g^{\prime}(z)\right|^{q}\left(1-|z|^{2}\right)^{q-2+s} \mathrm{~d} A(z)$ is an $s$-Carleson measure. Let $f \in B_{p}$. By Theorem 1 , we see that $I_{d}: B_{p}$ $\longrightarrow \mathscr{T}_{s, q(1-(1 / p))}^{q}\left(\mu_{g}\right)$ is bounded, i.e.,

$$
\begin{aligned}
& \sup _{I \subset \partial \mathscr{D}} \frac{1}{|I|^{s}(\log (2 /|I|))^{q(1-1 / p)}} \int_{S(I)}\left|\left(T_{g} f\right)^{\prime}(z)\right|^{q}\left(1-|z|^{2}\right)^{q-2+s} \mathrm{~d} A(z) \\
& \quad=\sup _{I \subset \partial \mathscr{D}} \frac{1}{|I|^{s}(\log (2 /|I|))^{q(1-1 / p)}} \int_{S(I)}|f(z)|^{q}\left|g^{\prime}(z)\right|^{q}\left(1-|z|^{2}\right)^{q-2+s} \mathrm{~d} A(z) \\
& \quad=\sup _{I \subset \partial \mathscr{D}} \frac{1}{|I|^{s}(\log (2 /|I|))^{q(1-1 / p)}} \int_{S(I)}|f(z)|^{q} \mathrm{~d} \mu_{g}(z) \leqslant\|f\|_{B_{p}}^{q}<\infty .
\end{aligned}
$$

By Lemma 3, we get that

$$
\sup _{a \in \mathscr{D}} \frac{1}{\left(\log \left(2 / 1-|a|^{2}\right)\right)^{q(1-(1 / p))}} \int_{\mathscr{D}}\left|\left(T_{g} f\right)^{\prime}(z)\right|^{q}\left(1-|z|^{2}\right)^{q-2}\left(1-\left|\sigma_{a}(z)\right|^{2}\right)^{s} \mathrm{~d} A(z)<\infty .
$$

Therefore, $\quad T_{g}: B_{p} \longrightarrow \mathscr{L} \mathscr{F}(q, q-2, s, q-(q / p)) \quad$ is bounded.

Next, we give an estimation for the essential norm of $T_{g}$. First, we recall some definitions. The essential norm of $T: X \longrightarrow Y$, denoted by $\|T\|_{e, X \longrightarrow Y}$, is defined by

$\|T\|_{e, X \longrightarrow Y}=\inf _{K}\left\{\|T-K\|_{X \longrightarrow Y}: K\right.$ is compact from $X$ to $\left.Y\right\}$.
Here, $X$ and $Y$ are Banach spaces, and $T: X \longrightarrow Y$ is a bounded linear operator. It is easy to see that $T: X \longrightarrow Y$ is compact if and only if $\|T\|_{e, X \rightarrow Y}=0$. Let $A$ be a closed subspace of $X$. Given $f \in X$, the distance from $f$ to $A$, denoted by $\operatorname{dist}_{X}(f, A)$, is defined by

$$
\operatorname{dist}_{X}(f, A)=\inf _{g \in A}\|f-g\|_{X} .
$$


Lemma 4. (see [14]). Let $1<q<\infty$ and $0<s<\infty$. If $g \in F(q, q-2, s)$, then

$$
\begin{gathered}
\operatorname{dist}_{F(q, q-2, s)}\left(g, F_{0}(q, q-2, s)\right) \approx \underset{r \longrightarrow 1^{-}}{\limsup }\left\|g-g_{r}\right\|_{F(q, q-2, s)} \\
\approx \limsup _{|a| \longrightarrow 1} \int_{\mathscr{D}}\left|g^{\prime}(z)\right|^{q}\left(1-|z|^{2}\right)^{q-2}\left(1-\left|\sigma_{a}(z)\right|^{2}\right)^{s} \mathrm{~d} A(z) .
\end{gathered}
$$

Here, $g_{r}(z)=g(r z), 0<r<1, z \in \mathscr{D}$.
Lemma 5. Let $1<p<q<\infty$ and $0<s<\infty$. If $0<r<1$ and $g \in F(q, q-2, s)$ then $T_{g_{r}}: B_{p} \longrightarrow \mathscr{L} \mathscr{F}(q, q-2, s, q-(q / p))$ is compact.

Proof. Given $\left\{f_{n}\right\} \subset B_{p}$ such that $\left\{f_{n}\right\}$ converges to zero uniformly on any compact subset of $\mathscr{D}$ and $\sup _{n}\left\|f_{n}\right\|_{B_{p}} \leq 1$. Since $g \in F(q, q-2, s) \subset \mathscr{B}$, we get that

$$
\left|g_{r}^{\prime}(z)\right| \lesssim \frac{\|g\|_{F(q, q-2, s)}}{1-r^{2}} .
$$

Hence,

$$
\begin{aligned}
& \left\|T_{g_{r}} f_{n}\right\|_{\mathscr{L} \mathscr{F}(q, q-2, s, q-(q / p))}^{q} \\
& \quad=\sup _{a \in \mathscr{D}} \frac{1}{\left(\log \left(2 / 1-|a|^{2}\right)\right)^{q(1-1 / p)}} \int_{\mathscr{D}}\left|f_{n}(z)\right|^{q}\left|g_{r}^{\prime}(z)\right|^{q}\left(1-|z|^{2}\right)^{q-2}\left(1-\left|\sigma_{a}((z))\right|^{2}\right)^{s} \mathrm{~d} A(z) \\
& \quad \leq \frac{\|g\|_{\mathscr{B}}^{q}}{\left(1-r^{2}\right)^{q}} \sup _{a \in \mathscr{D}} \int_{\mathscr{D}}\left|f_{n}(z)\right|^{q}\left(1-|z|^{2}\right)^{q-2}\left(1-\left|\sigma_{a}(z)\right|^{2}\right)^{s} \mathrm{~d} A(z) \\
& \quad \leq \frac{\|g\|_{F(q, q-2, s)}^{q}\left\|f_{n}\right\|_{B_{p}}^{q-p}}{\left(1-r^{2}\right)^{q}} \sup _{a \in \mathscr{D}} \int_{\mathscr{D}}\left|f_{n}(z)\right|^{p}\left(1-|z|^{2}\right)^{p-2}\left(1-\left|\sigma_{a}(z)\right|^{2}\right)^{s} \mathrm{~d} A(z) \\
& \quad \leq \frac{\|g\|_{F(q, q-2, s)}^{q}\left\|f_{n}\right\|_{B_{p}}^{q-p}}{\left(1-r^{2}\right)^{q}} \int_{\mathscr{D}}\left|f_{n}(z)\right|^{p}\left(1-|z|^{2}\right)^{p-2} \mathrm{~d} A(z) \\
& \quad \leq \frac{\|g\|_{F(q, q-2, s)}^{q}\left\|f_{n}\right\|_{B_{p}}^{q-p}}{\left(1-r^{2}\right)^{q}} \int_{\mathscr{D}}\left|f_{n}^{\prime}(z)\right|^{p}\left(1-|z|^{2}\right)^{p-2} \mathrm{~d} A(z) \\
& \quad \leqslant \frac{\|g\|_{F(q, q-2, s)}^{q}\left\|f_{n}\right\|_{B_{p}}^{q}}{\left(1-r^{2}\right)^{q}} .
\end{aligned}
$$

By the dominated convergence theorem, we get the desired result. The proof is completed.

Lemma 6. (see [23]). Let $X$ and $Y$ be two Banach spaces of analytic functions on $\mathscr{D}$. Suppose that

(1) The point evaluation functionals on $Y$ are continuous

(2) The closed unit ball of $X$ is a compact subset of $X$ in the topology of uniform convergence on compact sets

(3) $T: X \longrightarrow Y$ is continuous when $X$ and $Y$ are given the topology of uniform convergence on compact sets

Then, $T$ is a compact operator if and only if for any bounded sequence $\left\{f_{n}\right\}$ in $X$ such that $\left\{f_{n}\right\}$ converges to zero uniformly on every compact set of $\mathscr{D}$, the sequence $\left\{T f_{n}\right\}$ converges to zero in the norm of $Y$.

Theorem 4. Let $1<p<q<\infty$ and $0<s<\infty$. If $T_{g}: B_{p} \longrightarrow \mathscr{L} \mathscr{F}(q, q-2, s, q-(q / p))$ is bounded, then
$\left\|T_{g}\right\|_{e, B_{p} \longrightarrow \mathscr{L F}(q, q-2, s, q-(q / p))} \approx \operatorname{dist}_{F(q, q-2, s)}\left(g, F_{0}(q, q-2, s)\right)$.

Proof. Let $\left\{I_{n}\right\} \subseteq \partial \mathscr{D}$ and $\left|I_{n}\right| \longrightarrow 0$ as $n \longrightarrow \infty$. Suppose $e^{i \theta_{n}}$ is the center of $I_{n}$ and $w_{n}=\left(1-\left|I_{n}\right|\right) e^{i \theta_{n}}$. For each $n$, let

$$
f_{w_{n}}(z)=\left(\frac{1}{\log \left(2 / 1-\left|w_{n}\right|^{2}\right)}\right)^{1 / p} \log \frac{2}{1-\overline{w_{n}} z} .
$$

Then, $\left|f_{w_{n}}(z)\right| \approx\left(\log \left(2 /\left|I_{n}\right|\right)\right)^{1-1 / p}$ when $z \in S\left(I_{n}\right)$, and $\left\{f_{w_{n}}\right\}$ is bounded in $B_{p}$. Furthermore, $\left\{f_{w_{n}}\right\}$ converges to zero uniformly on every compact subset of $\mathscr{D}$. Given a compact operator $K: B_{p} \longrightarrow \mathscr{L} \mathscr{F}(q, q-2, s, q-(q / p))$, by Lemma 6, we have

$$
\lim _{n \longrightarrow \infty}\left\|K f_{w_{n}}\right\|_{\mathscr{L} \mathscr{F}(q, q-2, s, q-(q / p))}=0 .
$$

So, 


$$
\begin{aligned}
\left\|T_{g}-K\right\| & >\limsup _{n \longrightarrow \infty}\left\|\left(T_{g}-K\right) f_{w_{n}}\right\|_{\mathscr{L} \mathscr{F}(q, q-2, s, q-(q / p))} \\
& >\limsup _{n \longrightarrow \infty}\left(\left\|T_{g} f_{w_{n}}\right\|_{\mathscr{L} \mathscr{F}(q, q-2, s, q-(q / p))}-\left\|K f_{w_{n}}\right\|_{\mathscr{L} \mathscr{F}(q, q-2, s, q-(q / p))}\right) \\
& =\limsup _{n \longrightarrow \infty}\left\|T_{g} f_{w_{n}}\right\|_{\mathscr{L} \mathscr{F}(q, q-2, s, q-(q / p))} \\
& \geq \limsup _{n \longrightarrow \infty}\left(\frac{1}{\left(\log \left(2 / 1-\left|w_{n}\right|^{2}\right)\right)^{q(1-1 / p)}} \int_{\mathscr{D}}\left|f_{w_{n}}(z)\right|^{q}\left|g^{\prime}(z)\right|^{q}\left(1-|z|^{2}\right)^{q-2}\left(1-\left|\sigma_{w_{n}}(z)\right|^{2}\right)^{s} \mathrm{~d} A(z)\right)^{(1 / q)} \\
& \geq \limsup _{n \longrightarrow \infty}\left(\frac{1}{\left(\log \left(2 / 1-\left|w_{n}\right|^{2}\right)\right)^{q(1-1 / p)}} \int_{S\left(I_{n}\right)}\left|f_{w_{n}}(z)\right|^{q}\left|g^{\prime}(z)\right|^{q}\left(1-|z|^{2}\right)^{q-2}\left(1-\left|\sigma_{w_{n}}(z)\right|^{2}\right)^{s} \mathrm{~d} A(z)\right)^{(1 / q)} \\
& >\limsup _{n \longrightarrow \infty}\left(\frac{1}{\left|I_{n}\right|^{s}} \int_{S\left(I_{n}\right)}\left|g^{\prime}(z)\right|^{q}\left(1-|z|^{2}\right)^{q-2+s} \mathrm{~d} A(z)\right)^{(1 / q)},
\end{aligned}
$$

which implies that

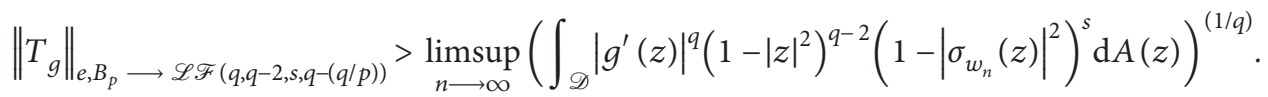

It follows from Lemma 4 that

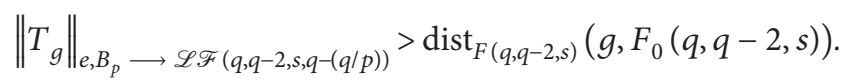

On the contrary, by Lemma $5, T_{g_{r}}: B_{p} \longrightarrow \mathscr{L} \mathscr{F}(q, q-$ $2, s, q-(q / p))$ is compact. Then,

$$
\begin{aligned}
\left\|T_{g}\right\|_{e, B_{p}} \longrightarrow \mathscr{L F}(q, q-2, s, q-(q / p)) & \leq\left\|T_{g}-T_{g_{r}}\right\|=\left\|T_{g-g_{r}}\right\| \\
& \approx\left\|g-g_{r}\right\|_{F(q, q-2, s)} .
\end{aligned}
$$

Using Lemma 4 again, we have

$$
\begin{aligned}
\left\|T_{g}\right\|_{e, B_{p}} \longrightarrow \mathscr{L F}(q, q-2, s, q-(q / p)) & \leq \limsup _{r \longrightarrow 1^{-}}\left\|g-g_{r}\right\|_{F(q, q-2, s)} \\
& \approx \operatorname{dist}_{F(q, q-2, s)}\left(g, F_{0}(q, q-2, s)\right) .
\end{aligned}
$$

The proof is completed.

The following result can be deduced by Theorem 4 directly.

Corollary 1. Let $1<p<q<\infty$ and $0<s<\infty$. If $T_{g}: B_{p} \longrightarrow \mathscr{L} \mathscr{F}(q, q-2, s, q-(q / p))$ is bounded, then $T_{g}:$ $B_{p} \longrightarrow \mathscr{L} \mathscr{F}(q, q-2, s, q-(q / p))$ is compact if and only if

$$
g \in F_{0}(q, q-2, s) .
$$

\section{Data Availability}

No data were used to support this study.

\section{Conflicts of Interest}

The authors declare that they have no conflicts of interest.

\section{Acknowledgments}

The first author was supported by the Science Foundation of Hanshan Normal University (no. XN202029). The third author was supported by NNSF of China (nos. 11801250 and 11871257), Overseas Scholarship Program for Elite Young and Middle-aged Teachers of Lingnan Normal University, Yanling Youqing Program of Lingnan Normal University (no. YL20200202), the Key Subject Program of Lingnan Normal University (nos. 1171518004 and LZ1905), and the Department of Education of Guangdong Province (no. 2018KTSCX133).

\section{References}

[1] R. Zhao, "On a general family of function spaces," Annales Academice Scientiarum Fennica, vol. 105, p. 56, 1996.

[2] J. Xiao, Holomorphic QClasses, Springer, Berlin, Germany, 2001.

[3] C. Pommerenke, "Schlichte Funktionen und analytische Funktionen von beschränkter mittlerer Oszillation," Commentarii Mathematici Helvetici, vol. 52, no. 1, pp. 591-602, 1977. 
[4] A. Aleman and A. Siskakis, "An integral operator on $H^{p}$," Complex Variables, Theory and Application, vol. 28, pp. 149-158, 1955.

[5] A. Aleman and A. Siskakis, "Integration operators on Bergman spaces," Indiana University Mathematics Journal, vol. 46, pp. 337-356, 1997.

[6] A. Aleman and J. A. Cima, "An integral operator onH p and Hardy's inequality," Journal d'Analyse Mathématique, vol. 85, no. 1, pp. 157-176, 2001.

[7] D. Girela and J. Á. Peláez, "Carleson measures, multipliers and integration operators for spaces of Dirichlet type," Journal of Functional Analysis, vol. 241, no. 1, pp. 334-358, 2006.

[8] P. Li, J. Liu, and Z. Lou, "Integral operators on analytic Morrey spaces," Science China Mathematics, vol. 57, no. 9, pp. 1961-1974, 2014.

[9] S. Li, J. Liu, and C. Yuan, "Embedding theorems for Dirichlet type spaces," Canadian Mathematical Bulletin, vol. 63, no. 1, 2019.

[10] S. Li and S. Stević, "Riemann-Stieltjes operators between $\alpha$ -Bloch spaces and Besov spaces," Mathematische Nachrichten, vol. 282, no. 6, pp. 899-911, 2009.

[11] S. Li and H. Wulan, "Volterra type operators on $Q_{K}$ spaces," Taiwanese Journal of Mathematics, vol. 14, pp. 195-211, 2010.

[12] J. Pau and R. Zhao, "Carleson measures, Riemann-Stieltjes and multiplication operators on a general family of function spaces," Integral Equations and Operator Theory, vol. 78, no. 4, pp. 483-514, 2014.

[13] R. Qian and S. Li, "Volterra type operators on Morrey type spaces," Mathematical Inequalities \& Applications, vol. 18, no. 4, pp. 1589-1599, 2015.

[14] R. Qian and S. Li, "Embedding of Besov spaces into tent spaces and applications," Journal of Contemporary Mathematical Analysis, vol. 6, no. 1, 2021.

[15] R. Qian and X. Zhu, "Embedding of $Q_{s}$ spaces into tent spaces and Volterra integral operator," AIMS Mathematics, vol. 6, pp. 698-711, 2021.

[16] Y. Shi and S. Li, "Essential norm of integral operators on Morrey type spaces," Mathematical Inequalities \& Applications, vol. 19, pp. 385-393, 2016.

[17] A. G. Siskakis and R. Zhao, "A volterra type operator on spaces of analytic functions," Function Spaces, vol. 232, pp. 299-311, 1999.

[18] J. Xiao, "The Qp Carleson measure problem," Advances in Mathematics, vol. 217, no. 5, pp. 2075-2088, 2008.

[19] L. Carleson, "Interpolations by bounded analytic functions and the Corona problem," The Annals of Mathematics, vol. 76, no. 3, pp. 547-559, 1962.

[20] N. Arcozzi, R. Rochberg, and E. Sawyer, "Carleson measures for analytic Besov spaces," Revista Matemática Iberoamericana, vol. 18, pp. 443-510, 2002.

[21] J. Liu, Z. Lou, and K. Zhu, "Embedding of Möbius invariant function spaces into tent spaces," The Journal of Geometric Analysis, vol. 27, no. 2, pp. 1013-1028, 2017.

[22] K. Zhu, "Operator theory in function spaces," in Mathematical Surveys and Monographs, C. B Huijsmans, Ed., Springer, Berlin, Germany, 2007.

[23] M. Tjani, "Compact composition operators on some möbius invariant banach spaces," Ph.D Dissertation, Michigan State University, East Lansing, Michigan, 1996. 